\title{
Síndrome de Burnout em residentes multiprofissionais em saúde
}

\section{Burnout syndrome in multi-professional healthcare residents}

Síndrome de Burnout en residentes multiprofesionales en salud

\author{
Douglas de Souza e Silva'; Magno Conceição das Merces"'; Marcio Costa de Souzalll; \\ Antônio Marcos Tosoli Gomes'v; Sueli Bonfim Lago i ; Mônica Beltrame ${ }^{\text {IV }}$
}

\begin{abstract}
RESUMO
Objetivo: estimar a prevalência da Síndrome de Burnout e identificar o perfil sociodemográfico, laboral, estilo de vida e saúde de residentes multiprofissionais de saúde de uma Universidade pública da Bahia, Brasil. Metodologia: estudo transversal, descritivo, realizado com 63 residentes. Utilizou-se o Maslach Burnout Inventory e um questionário contendo questões sociodemográficas, laborais, estilo de vida e saúde. Resultados: ao analisar as dimensões da síndrome, $82,5 \%$ apresentaram nível alto de exaustão emocional; 55,5\% nível moderado de despersonalização e $88,8 \%$ nível alto de reduzida realização profissional. Destaca-se a maior ocorrência do Burnout em enfermeiros (50\%), profissionais atuantes no âmbito hospitalar (71,4\%) e em recém-formados (média 2,1 anos). 60,3\% dos residentes consideraram não ter uma alimentação saudável e 29,5\% apresentaram excesso de peso. Conclusão: os resultados apontam uma prevalência significativa de síndrome de burnout, sendo encontradas altas alterações nas três dimensões da síndrome em 22,2\% dos residentes.

Descritores: Educação de pós-graduação; estresse ocupacional; esgotamento profissional; saúde do trabalhador.
\end{abstract}

\section{ABSTRACT}

Objective: to estimate the prevalence of Burnout Syndrome, and identify the sociodemographic, occupational, lifestyle, and health profile of multi-professional healthcare residents at a public university in Bahia, Brazil. Methods: a descriptive, crosssectional study was conducted with 63 residents, using the Maslach Burnout Inventory and a questionnaire on sociodemographic, labor, lifestyle, and health issues. Results: on examining the dimensions of the syndrome, $82.5 \%$ were found to present high levels of emotional exhaustion; $55.5 \%$, moderate levels of depersonalization; and $88.8 \%$, high levels of diminished professional fulfilment. Burnout occurred most often in nurses (50\%), professionals working in hospitals $(71.4 \%)$ and recent graduates (mean 2.1 years), while $60.3 \%$ of the residents regarded their diet at unhealthy, and $29.5 \%$ were overweight. Conclusion: the results indicate a significant prevalence of Burnout Syndrome, and marked changes in the three dimensions of the syndrome were found in $22.2 \%$ of the residents.

Descriptors: Education graduate; occupational stress; burnout professional; occupational health.

\section{RESUMEN}

Objetivo: estimar la prevalencia del Síndrome de Burnout e identificar el perfil sociodemográfico, laboral, estilo de vida y salud de residentes multiprofesionales de salud de una universidad pública de Bahía, Brasil. Método: estudio transversal, descriptivo, realizado junto a 63 residentes. Se utilizó el Maslach Burnout Inventory y un cuestionario que contenía cuestiones sociodemográficas, laborales, estilo de vida y salud. Resultados: al analizar las dimensiones del síndrome, el 82,5\% presentó un alto nivel de agotamiento emocional; $55,5 \%$ nivel moderado de despersonalización y $88,8 \%$ nivel alto de reducida realización profesional. Se destaca la mayor ocurrencia de Burnout en enfermeros (50\%), profesionales actuantes en el ámbito hospitalario $(71,4 \%$ y recién graduados (promedio 2,1 años). El 60,3\% de los residentes consideró no tener una alimentación sana y el $29,5 \%$ presentó un exceso de peso. Conclusión: los resultados apuntan a una prevalencia significativa de síndrome de Burnout y se encontraron grandes alteraciones en las tres dimensiones del síndrome en el $22,2 \%$ de los residentes. Descriptores: Educación de postgrado; estrés ocupacional; agotamiento profesional; salud del trabajador.

\section{INTRODUÇÃO}

Sabe-se que o trabalho pode induzir ao adoecimento, podendo o estresse ser um fator causal para doenças. Define-se estresse como uma reação desencadeada no organismo, por um estímulo visto como ameaçador, no qual excede as capacidades adaptativas do indivíduo, onde há ativação de manifestações neuroendócrinas com vista ao enfrentamento e equilíbrio homeostático. A intensidade do estresse e as formas de enfrentamento podem levar ao surgimento de problemas à saúde, como a Síndrome de Burnout (SB) ${ }^{1,2}$.

'Enfermeiro. Especialista em Terapia Intensiva sob o formato de residência, Mestrando do Programa de Pós-graduação em Ciências da Saúde, Universidade Federal da Bahia. Brasil. E-mail: douglasss-gbi@hotmail.com

"Enfermeiro. Mestre em Saúde Coletiva. Professor da Universidade do Estado da Bahia. Brasil. E-mail: mmerces@uneb.br

I'Fisioterapeuta. Mestre em Saúde Coletiva. Professor da Universidade do Estado da Bahia. Brasil. e-mail: mcsouza@uneb.br

IVEnfermeiro. Doutor em Enfermagem. Professor Titular, Universidade do Estado do Rio de Janeiro. Brasil. E-mail: mtosoli@gmail.com

vGraduada em Filosofia. Mestra em Filosofia. Professora da Universidade do Estado da Bahia. Brasil. E-mail: slpinheiro@uneb.br

v'Cirurgiã Dentista. Doutora em Radiologia. Professora da Universidade do Estado da Bahia. Brasil. E-mail: mbeltrame@uneb.br 
Nas atividades de formação profissional, tanto nos cursos de graduação, como nos programas de pós-graduação, há situações aviltantes que podem ser avaliadas como estressoras. Frente ao cenário da pós-graduação, destacam-se os Programas de Residência Multiprofissional em Saúde, enquadrados como especialização (lato sensu), que surgiram no Brasil frente à promulgação da Lei $\mathrm{n}^{\circ} 11.129$ de 2005, e da portaria interministerial no 1.117 de 2005, instituída no âmbito do Ministério da Saúde e da Educação, tendo como escopo formar e qualificar profissionais de saúde, não médicos, no Sistema Único de Saúde (SUS), e como estratégia o ensino/aprendizado e a formação em serviço, mediante acompanhamento e supervisão ${ }^{3}$.

Os residentes da área de saúde são expostos a riscos ocupacionais e conseguintemente a alto grau de estresse laboral. Muitos são os preditores para tal questão, certamente, devido às responsabilidades profissionais, cumprimento de pautas solicitadas pelas supervisões e coordenação do programa de residência, jornada de trabalho exaustiva, além de rotinas de estudos necessários para a construção do conhecimento na área de atuação. Estas situações desafiadoras provocam sensações de tensão, ansiedade e medo, que podem ser avaliadas como estressoras e interferir diretamente na qualidade de vida e ao surgimento da Síndrome de Burnout, sendo esta, decorrente do estresse ocupacional ${ }^{4}$.

Diante da contextualização apresentada, registra-se a escassa produção científica que versam sobre a saúde mental dos residentes multiprofissionais, tendo maior ênfase publicações sobre o burnout em profissionais das residências médicas. Estudos sobre burnout em residentes de medicina apontam prevalências significativas da síndrome ${ }^{5,6}$. Salienta-se que recentemente a organização mundial de saúde incluiu o burnout na classificação internacional de doenças em sua 11ạ edição, como um fenômeno ligado ao trabalho.

Nesta perspectiva, levantou-se como questão de pesquisa: Qual a ocorrência da Síndrome de Burnout em residentes multiprofissionais de saúde? Desse modo, objetivou-se estimar a prevalência da Síndrome de Burnout e identificar o perfil sociodemográfico, laboral, estilo de vida e saúde dos residentes multiprofissionais em saúde.

\section{REFERENCIAL TEÓRICO}

Assim, a SB, decorre da cronificação do estresse laboral, mediante um prolongado processo de tentativas de lidar com determinadas condições de estresse, e caracteriza-se pela presença de altos níveis de Exaustão Emocional (EE), Despersonalização (DP) e Redução da Realização Profissional (RRP). Considera-se a síndrome como um quadro clínico mental extremo do estresse ocupacional, implicando em efeitos deletérios a saúde do trabalhador ${ }^{7-9}$.

Uma das principais descrições da SB foi realizada na década de 70 por Freudenberger em seu artigo Staff Burnout, datado de 1974, onde alerta a comunidade científica dos problemas a que os profissionais de saúde estão expostos em função de seu trabalho ${ }^{10}$. O burnout apresenta uma série de consequências de natureza emocional, física e social/comportamental, tais como: (i) insatisfação profissional (frustação e sentimentos de incompetência), (ii) dores, insônia, excesso de peso, (iii) absenteísmo, isolamento social e problemas alimentares ${ }^{11,12}$. Contribui para um impacto negativo na saúde mental e física dos trabalhadores ${ }^{13,14}$.

Sobre as dimensões do burnout, a EE apresenta sentimentos de esgotamento físico, mental, emocional, na maioria das vezes devido à sobrecarga; na DP, observam-se condutas e resposta negativas a colegas e pacientes, estando o cinismo e o isolamento presentes. Não significa que o individuo deixou de ter personalidade, mas, que esta sofreu ou sofre alterações, levando o profissional a um contato frio e impessoal; já a RRP se constata que o profissional não se sente competente e realizado em sua função, apresentando baixa autoestima, desmotivação e por vezes ímpetos de abandonar o emprego ${ }^{15,16}$.

\section{METODOLOGIA}

Estudo transversal, de caráter descritivo, realizado com residentes multiprofissionais em saúde vinculados a uma Universidade Pública, localizada no estado da Bahia, região nordeste do Brasil. A coleta de dados se deu no período de julho a novembro do ano de 2018.

Foram convidados para participar do presente estudo, um total de 74 (100\%) residentes do primeiro e segundo ano. Foram excluídos os profissionais residentes que não assinaram o Termo de Consentimento Livre e Esclarecido (TCLE) após explicação da pesquisa, $02(2,7 \%)$, que realizou tratamento psiquiátrico $04(5,4 \%)$ e no período de ciclo gravídico puerperal 01 (1,3\%); ocorreram $04(5,4 \%)$ recursas. A população final da pesquisa foi constituída por 63 residentes multiprofissionais em saúde, obtendo-se uma taxa de resposta de $94 \%$.

Foi elaborado um questionário composto por questões relacionadas às condições sociodemográficas, laborais, estilo de vida e saúde. A Síndrome de Burnout foi avaliada pelo inventário Maslach Burnout Inventory (MBI) - Human 
Services Surve ${ }^{17}$, e dicotomizada conforme critério de Ramirez et al. (1996), como presente ou ausente, ao considerar a existência de altos escores nas dimensões de EE e DP e baixos escores na RRP ${ }^{18}$.

Obtidos os escores das pontuações do $\mathrm{MBI}$, institui-se os pontos de corte para cada dimensão, classificadas em níveis alto, moderado e baixo. Para a exaustão emocional (EE): alto ( $\geq 27$ pontos), moderado (19 a 26 pontos) e baixo ( $\leq 18$ pontos); reduzida realização profissional (RRP): alto ( $\leq 33$ pontos), moderado (34 a 39 pontos) e baixo ( $\geq 40$ pontos); despersonalização (DP): alto ( $\geq 10$ pontos), moderado ( 6 a 9 pontos) e baixo ( $\leq 5$ pontos).

Avaliou-se a confiabilidade interna das categorias do instrumento $\mathrm{MBI}$ utilizando o coeficiente alfa de cronbach. Os valores de alfa acima de 0,70 indicam aceitável consistência interna, sustentando a confiabilidade do instrumento, sendo 0,82 para EE, 0,79 para DP e 0,81 para RRP.

Realizou-se a mensuração da circunferência abdominal e sua avaliação se deu através dos seguintes pontos de corte: normal ( $<88 \mathrm{~cm}$ para mulheres e $<102 \mathrm{~cm}$ para os homens) e de risco elevado ( $\geq 88 \mathrm{~cm}$ para as mulheres e $\geq 102$ para os homens). Para verificação do excesso de peso foi utilizado o índice de massa corpórea (IMC) e classificado de acordo a Organização Mundial de Saúde (OMS) em baixo peso (IMC $<18,5 \mathrm{~kg} / \mathrm{m}^{2}$ ), eutrófico $\left(\mathrm{IMC}<25,0 \mathrm{~kg} / \mathrm{m}^{2}\right)$ e excesso de peso $\left(\mathrm{IMC}>\text { a } 25,0 \mathrm{~kg} / \mathrm{m}^{2}\right)^{19}$.

As análises dos dados foram realizadas com auxílio do software Statistic Package for Social Sciences (SPSS) ${ }^{\circledR}$ versão 22 para Windows $^{\circledR}$ na Universidade do Estado da Bahia (UNEB). Realizou-se a análise descritiva para caracterização da população e calculadas as frequências absolutas e relativas das variáveis de interesse.

O desenvolvimento do estudo atendeu as normas nacionais de ética em pesquisa envolvendo seres humanos, em consonância com a resolução no 466/2012. O projeto foi submetido ao Comitê de Ética em Pesquisa com Seres Humanos da UNEB, sendo aprovado pelo parecer número 2.724.131.

\section{RESULTADOS}

A população do estudo correspondeu a 63 (100\%) residentes multiprofissionais, destes, 31 (49,2\%) pertenciam ao primeiro ano de curso e $32(50,8 \%)$ ao segundo ano, lotados nos seguintes núcleos de atuação: Saúde da Família 26 (41,3\%), Terapia Intensiva $13(20,6 \%)$, Nutrição Clínica $9(14,3 \%)$, Oncologia $15(23,8 \%)$. No tocante às categorias profissionais 25 (39,7\%) eram enfermeiros (as), 7 (11,1\%) nutricionistas, 7 (11,1\%) farmacêuticos (as), 7 (11,1\%) psicólogas; 6 (9,5\%) fisioterapeutas; 6 (9,5\%) fonoaudiólogas; 4 (6,3\%) cirurgiã dentista e 1 (1,6\%) assistente social, de acordo com a Tabela 1.

As características sociodemográficas dos profissionais evidenciaram uma população jovem (79,3\%) com média de 26,7 anos (DP $\pm 2,9)$, predominantemente do sexo feminino $(92,1 \%)$, negros (as) $(79,3 \%)$, solteiros (as) $(85,7 \%)$ e de religião católica (36,5\%). No tocante as características laborais, $71,4 \%$ relataram satisfação com a ocupação atual, todavia, $65,1 \%$ estavam insatisfeitos com sua situação econômica. 93,7\% consideraram a residência estressante. Quando questionados sobre assédio moral, 69,8\% sentiram-se assediados por preceptores ou profissionais do serviço e $19 \%$ por pacientes. $71,4 \%$ dos residentes informaram ter pausa de descanso no campo de estágio/trabalho, conforme Tabela 1.

Sobre as características de estilo de vida e saúde, 52,3\% dos residentes não praticavam exercícios físicos rotineiramente durante a semana; $98,4 \%$ não eram tabagistas e 77,8\% consumiam bebida alcoólica. 57,1\% relataram padrão de sono de 6 a 8 horas por dia e 39,7\% até 5 horas por dia. A presença de dores nas costas ao final de um dia de trabalho foi relatada por $76,2 \%$. A presença de dores de cabeça e problemas digestivos foram mencionados por $73 \%$ e $54 \%$ dos residentes, respectivamente. 60,3\% informaram não dispor de hábitos de alimentação saudável, deste modo, o excesso de peso representado pelo IMC estava presente em $29,5 \%$. Os achados da medida de circunferência da cintura revelaram uma prevalência de adiposidade abdominal de 5,3\%, ressaltando a ausência nos profissionais do sexo masculino, apresentado na Tabela 2.

Ao distribuir os níveis de cada dimensão do $M B I$ entre os residentes multiprofissionais em saúde, pôde-se observar que $82,5 \%$ apresentaram nível alto de exaustão emocional (EE); 55,5\% pontuaram nível moderado de despersonalização (DP); e na reduzida realização profissional (RRP), 88,8\% apresentaram nível alto. Ao analisar as médias de cada dimensão, verificou-se uma alta EE, moderada DP e alta RRP, conforme a Tabela 3.

Ao verificar a SB, a prevalência apresentada foi de 14 (22,2\%), indicando níveis altos nas três dimensões da SB, alertando para o desenvolvimento da síndrome. Dos que apresentaram indicativos da SB (22,2\%), 50\% eram enfermeiros (as), 14,3\% nutricionistas, e, com 7,1\% cada, estavam inclusos fisioterapeutas, fonoaudiólogos (as), farmacêuticos (as), psicólogos (as) e cirurgião (a) dentista. Os residentes do núcleo de terapia intensiva foram os mais acometidos $(42,9 \%)$, seguido de saúde da família $(28,6 \%)$, oncologia $(14,3 \%)$ e nutrição clínicas $(14,3 \%)$, assim, os 
profissionais atuantes no âmbito hospitalar pontuaram 71,4\%. A média de tempo de formação foi de 2,1 anos, com predominância de mulheres (85,7\%), e negros (as) (92,8\%), de acordo com a Tabela 3.

TABELA 1: Características sociodemográficas, econômicas e laborais de Residentes

Multiprofissionais em Saúde. Salvador, Bahia, Brasil, 2018 (N=63)

\begin{tabular}{|c|c|c|}
\hline Variáveis & $\mathbf{N}$ & $\%$ \\
\hline \multicolumn{3}{|l|}{ Idade } \\
\hline Até 30 anos & 50 & 79,3 \\
\hline Maior que 30 anos & 13 & 20,7 \\
\hline \multicolumn{3}{|l|}{ Sexo } \\
\hline Feminino & 58 & 92,1 \\
\hline Masculino & 5 & 7,9 \\
\hline \multicolumn{3}{|l|}{ Raça/cor } \\
\hline Negros(a) & 50 & 79,3 \\
\hline Não negros(a) & 13 & 20,7 \\
\hline \multicolumn{3}{|l|}{ Estado Civil } \\
\hline Solteiro(a) & 54 & 85,7 \\
\hline Casado(a) & 9 & 14,3 \\
\hline \multicolumn{3}{|l|}{ Religião } \\
\hline Católica & 23 & 36,5 \\
\hline Evangélica & 12 & 19,0 \\
\hline Espírita & 9 & 14,3 \\
\hline Matriz Africana & 1 & 1,6 \\
\hline Outras & 18 & 28,6 \\
\hline \multicolumn{3}{|l|}{ Categoria Profissional } \\
\hline Enfermeiro(a) & 25 & 39,7 \\
\hline Fisioterapeuta & 6 & 9,5 \\
\hline Fonoaudiólogo(a) & 6 & 9,5 \\
\hline Nutricionista & 7 & 11,1 \\
\hline Farmacêutico(a) & 7 & 11,1 \\
\hline Psicológo(a) & 7 & 11,1 \\
\hline Assistente Social & 1 & 1,6 \\
\hline Cirurgião(a) Destista & 4 & 6,4 \\
\hline \multicolumn{3}{|l|}{ Núcleo de Atuação } \\
\hline Saúde da Família & 26 & 41,3 \\
\hline Terapia Intensiva & 13 & 20,6 \\
\hline Oncologia & 15 & 23,8 \\
\hline Nutrição Clínica & 9 & 14,3 \\
\hline \multicolumn{3}{|l|}{ Ano da Residência } \\
\hline Primeiro Ano & 31 & 49,2 \\
\hline Segundo Ano & 32 & 50,8 \\
\hline \multicolumn{3}{|l|}{ Situação Econômica } \\
\hline Satisfeita & 22 & 34,9 \\
\hline Insatisfeita & 41 & 65,1 \\
\hline \multicolumn{3}{|l|}{ Satisfação com Ocupação Atual } \\
\hline Sim & 45 & 71,4 \\
\hline Não & 18 & 28,6 \\
\hline \multicolumn{3}{|c|}{ Considera a Residência Estressante } \\
\hline Sim & 59 & 93,7 \\
\hline Não & 4 & 6,3 \\
\hline \multicolumn{3}{|c|}{ Assédio de Preceptor ou Profissional do Serviço } \\
\hline Sim & 44 & 69,8 \\
\hline Não & 19 & 30,2 \\
\hline \multicolumn{3}{|l|}{ Assédio de Pacientes } \\
\hline Sim & 12 & 19,0 \\
\hline Não & 51 & 81,0 \\
\hline \multicolumn{3}{|c|}{ Pausa para Descanso Durante Trabalho } \\
\hline Sim & 45 & 71,4 \\
\hline Não & 18 & 28,6 \\
\hline
\end{tabular}


TABELA 2: Características de estilo de vida e saúde de Residentes Multiprofissionais em Saúde. Salvador, Bahia, Brasil, 2018. (N=63)

\begin{tabular}{|c|c|c|}
\hline Variáveis & $\mathbf{N}$ & $\%$ \\
\hline \multicolumn{3}{|l|}{ Faz Exercícios Físicos Regularmente } \\
\hline Sim & 30 & 47,7 \\
\hline Não & 33 & 52,3 \\
\hline \multicolumn{3}{|l|}{ Tabagista } \\
\hline Sim & 1 & 1,6 \\
\hline Não & 62 & 98,4 \\
\hline \multicolumn{3}{|l|}{ Consumo de Bebida Alcoólica } \\
\hline Sim & 49 & 77,8 \\
\hline Não & 14 & 22,2 \\
\hline \multicolumn{3}{|l|}{ Padrão de Sono } \\
\hline Até 5 horas dia & 25 & 39,7 \\
\hline 6 a 8 horas dia & 36 & 57,1 \\
\hline Maior que 8 horas dia & 2 & 3,2 \\
\hline \multicolumn{3}{|c|}{ Necessidade de Acompanhamento Psicológico** } \\
\hline Sim & 21 & 33,3 \\
\hline Não & 42 & 66,7 \\
\hline \multicolumn{3}{|c|}{ Dores nas Costas ao Final do dia de Trabalho** } \\
\hline Sim & 48 & 76,2 \\
\hline Não & 15 & 23,8 \\
\hline \multicolumn{3}{|l|}{ Dores de Cabeça** } \\
\hline Sim & 46 & 73,0 \\
\hline Não & 17 & 27,0 \\
\hline \multicolumn{3}{|l|}{ Tonturas** } \\
\hline Sim & 23 & 36,5 \\
\hline Não & 40 & 63,5 \\
\hline \multicolumn{3}{|l|}{ Tremores** } \\
\hline Sim & 11 & 17,5 \\
\hline Não & 52 & 82,5 \\
\hline \multicolumn{3}{|l|}{ Falta de $\mathrm{Ar}^{* *}$} \\
\hline Sim & 20 & 31,7 \\
\hline Não & 43 & 68,3 \\
\hline \multicolumn{3}{|l|}{ Problemas Digestivos** } \\
\hline Sim & 34 & 54,0 \\
\hline Não & 29 & 46,0 \\
\hline \multicolumn{3}{|c|}{ Considera a Alimentação Saudável** } \\
\hline Sim & 25 & 39,7 \\
\hline Não & 38 & 60,3 \\
\hline \multicolumn{3}{|l|}{$\mathrm{IMC}\left(\mathrm{kg} / \mathrm{m}^{2}\right)^{*}$} \\
\hline Eutrofismo & 39 & 64,0 \\
\hline Baixo Peso & 4 & 6,5 \\
\hline Excesso de Peso & 18 & 29,5 \\
\hline \multicolumn{3}{|l|}{ Circunferência de Cintura (cm) } \\
\hline \multicolumn{3}{|l|}{ Mulheres } \\
\hline$\geq 88$ & 3 & 5,8 \\
\hline$<88$ & 49 & 94,2 \\
\hline \multicolumn{3}{|l|}{ Homens } \\
\hline$\geq 102$ & 0 & 0,0 \\
\hline$<102$ & 5 & 100 \\
\hline \multicolumn{3}{|l|}{ Adiposidade Abdominal } \\
\hline Presente & 3 & 5,3 \\
\hline Ausente & 54 & 94,7 \\
\hline
\end{tabular}

*Variável com dados perdidos **Dados auto referidos 
TABELA 3: Distribuição dos resultados Maslach Burnout Inventory entre os Residentes Multiprofissionais em Saúde. Salvador, Bahia, Brasil, 2018. ( $\mathrm{N}=63$ )

\begin{tabular}{|c|c|c|c|c|c|c|}
\hline \multirow[t]{2}{*}{ Dimensões } & \multicolumn{3}{|c|}{ Níveis N (\%) } & \multirow{2}{*}{$\begin{array}{c}\text { Média de } \\
\text { Pontos }\end{array}$} & \multirow{2}{*}{$\begin{array}{l}\text { Desvio } \\
\text { Padrão }\end{array}$} & \multirow[t]{2}{*}{ Amplitude } \\
\hline & Baixo & Moderado & Alto & & & \\
\hline Exaustão Emocional & $01(1,6)$ & $10(15,9)$ & $52(82,5)$ & 30,7 & 4,99 & $26(16-42)$ \\
\hline Despersonalização & $10(16)$ & $35(55,5)$ & $18(28,5)$ & 8,4 & 2,85 & $13(5-18)$ \\
\hline Reduzida Realização Profissional & - & $07(11,2)$ & $56(88,8)$ & 28,3 & 3,60 & $08(22-30)$ \\
\hline
\end{tabular}

\section{DISCUSSÃO}

A saúde mental e ocupacional dos profissionais em saúde e em destaque os residentes multiprofissionais é uma assunto de grande relevância na sociedade atual. Evidentemente que não só para os profissionais de saúde a saúde mental é importante, mas, em se tratando de um campo profissional ligado especificamente ao cuidado, estar bem do ponto de vista mental e físico é extremamente importante. Fatores diversos contribuem para que um indivíduo possa "estar bem" consigo e com os outros, no entanto, advoga-se aqui, que o trabalho possa ser uma questão central à vida e influencie diretamente nas questões pessoais, sociais ${ }^{20}$.

Na verdade, o trabalho é a forma de inserção social e só pode ser entendido como trabalho social. Nesse cenário, é plausível de pontuação e reflexão o cenário complexo que se vivencia no mundo do trabalho, destaque para as questões de precarização generalizada do trabalho, a terceirização, a sobrecarga de funções, as relações intreprofissionais complexas, jornadas excessivas nos quais muitos profissionais estão inseridos. Certamente, questões herdadas do modelo capitalista de produção ${ }^{21}$.

O equilíbrio da saúde mental do trabalhador da saúde é fundamental para que haja uma assistência digna, segura, e medidas para a prevenção do burnout devem ser pensadas, visto que, esta síndrome relaciona-se exclusivamente com o labor gerando consequências pessoais e institucionais, como grandes casos de presenteísmo, absenteísmo e afastamentos ${ }^{13,14}$.

As características sociodemográficas de todos os profissionais aqui apresentados evidenciaram uma população jovem, predominantemente do sexo feminino, negros e solteiros. $71,4 \%$ relataram satisfação com a ocupação atual, todavia, $65,1 \%$ estavam insatisfeitos com sua situação econômica e $93,7 \%$ consideram a residência estressante. Em um estudo que objetivou descrever o perfil sociodemográfico, profissional e acadêmico de residentes multiprofissionais de um hospital universitário, obteve achados semelhantes, apontando a predominância do sexo feminino, solteiros, sem filhos, na faixa etária entre 25 e 29 anos, atuantes na área hospitalar ${ }^{22}$.

Autores enfatizam que durante o período da residência há redução da qualidade de vida, relacionando-a as características da formação em serviço, tais como; extensa carga horária semanal, assistência a pacientes críticos e privação do sono. A residência é um período no qual o profissional é submetido a desgastes, podendo prejudicar o seu aprendizado e a sua saúde, além de colocar em risco a segurança do paciente ${ }^{23,24}$. As consequências do burnout são deletérias aos profissionais de saúde, pacientes e instituições de saúde, pois podem estar relacionadas as iatrogenias e condições adversas à segurança do paciente ${ }^{25}$.

Ressalta-se que a residência multiprofissional é uma modalidade de ensino de pós-graduação lato sensu com uma carga horária de 60 horas semanais e tem como base o ensino em serviço e duração de dois anos. Fatores como a extensa carga horária de trabalho, a junção de ser pós-graduando e trabalhador e a pouca experiência profissional prévia na área de formação da residência, são fatores predisponentes ao adoecimento ${ }^{26}$.

A prevalência apresentada no presente estudo da SB foi de $22,2 \%$, não diferindo de outros estudos nacionais. Tavares e colaboradores encontraram uma prevalência de $20,8 \%$, com predominância nos indivíduos jovens, do gênero feminino, solteiros, recém-formados e inseridos nos setores de alta complexidade ${ }^{27}$.

Estudo com residentes multiprofissionais encontrou prevalência de $27 \%$, mais prevalente entre os jovens e enfermeiros (as). A alta EE correspondeu a $37,8 \%$ e RRP 48,6\%, aproximando-se parcialmente dos resultados encontrados no presente estudo ${ }^{26}$. A discrepância apresentada na EE e RRP pode ser explicada pelos critérios metodológicos utilizados na pesquisa de referência citada ${ }^{28}$. 
O presente estudo aponta que os residentes com indicativos da SB são profissionais jovens, recém-formados, com predominância do sexo feminino e atuantes nos ambientes hospitalares, na média e alta complexidade. Estudos corroboram com esses achados e enfatizam também, que os residentes acumulam atividades acadêmicas variadas, como seminários, provas, trabalhos de conclusão de curso que demandam tempo e que as horas destinadas às atividades práticas são maiores do que as destinadas às atividades acadêmicas. $O$ que se constata é que esse acúmulo de atividades pode ser avaliado como fatores desencadeadores de estresse laboral e caso o profissional não atente para buscar medidas de enfrentamento a estes fatores, certamente pode leva-lo ao burnout ${ }^{4,24}$.

Um dado alarmante pontuado, diz respeito ao percentual de residentes que informaram a ocorrência de assédio moral por parte dos preceptores ou funcionários do serviço onde realizam as práticas laborais. 0 assédio moral é caracterizado pela exposição dos trabalhadores a situações constrangedoras e humilhantes de forma repetitiva, através de relações hierárquicas autoritárias. Assim, esse tipo de conduta pode incidir no processo saúde-doença, levando a consequências físicas e psicológicas, formação de conflitos, falta de confiança, absenteísmo, isolamento e desistência do vínculo laboral ${ }^{29}$.

Os estressores inerentes a cada profissão, atrelados aos advindos com a formação profissional que a residência multiprofissional proporciona, expõem os residentes à possibilidade de adquirir a SB. Dentre os múltiplos fatores desencadeadores da síndrome, destacam-se a insatisfação quanto à substituição de funcionários do setor, desvio de função, baixa remuneração, esgotamento físico, mental/emocional, a diminuição de tempo para o lazer e, principalmente a conflituosa relação de falta de identidade profissional. Danos esses que podem interferir nas relações familiares, institucionais e sociais. Sugere-se a necessidade de promover atividades educativas e de orientação sobre estresse e a promoção de estratégias de enfrentamento ${ }^{30-32}$.

No tocante as características de estilo de vida e biologia humana verificou-se que $52,3 \%$ dos residentes referiram a não prática de exercícios físicos regularmente durante sua rotina semanal, além de não dispor de uma alimentação saudável, apontada por $60,3 \%$. Neste contexto, verifica-se que $29,5 \%$ encontravam-se com excesso de peso, tendo seu IMC maior que $25 \mathrm{~kg} / \mathrm{m}^{2}$. Apesar da pequena prevalência de adiposidade abdominal encontrada no estudo (5,3\%), há na literatura estudos que apontam a plausibilidade biológica e uma associação entre o estresse e consequentemente o burnout com o aumento da adiposidade abdominal e ganho de peso ${ }^{33}$. Possivelmente existe uma relação entre o estresse e consequentemente a SB com o aumento de peso e adiposidade abdominal, levando a ingesta de alimentos calóricos e a estimulação do eixo Hipotálamo-Hipófise-Adrenal (HHA) e como consequência a produção de cortisol que se relaciona à obesidade visceral visando aumentar a quantidade de energia disponível para as situações de enfrentamento ao estresse ${ }^{33-35}$.

Enfatiza-se que no processo de formação profissional no qual o residente encontra-se inserido há possibilidade de desenvolvimento de atividades de caráter multiprofissional e consequentemente maior oportunidade de aprendizagem, sendo um período de excelente troca de conhecimento e crescimento profissional, capacitando-os profissionalmente na sua área de formação, sendo este profissional habilitado para futuras experiências profissionais com domínio e conhecimento prático e científico.

A principal limitação do estudo se baseia ao delineamento do estudo transversal que impossibilita o estabelecimento de relações causais e da presença de dados auto referidos. Estudos que analisem a saúde mental e ocupacional dos residentes em saúde são de suma importância, visto aos fatores relacionados ao estresse laboral e consequentemente ao adoecimento físico e mental.

\section{CONCLUSÃO}

Os resultados apontam uma prevalência significativa de síndrome de burnout, com maior ocorrência em Enfermeiros (as) e em profissionais atuantes nos setores hospitalares. Os profissionais acometidos eram recémformados, com predominância do gênero feminino, e negros (as). Destaca-se a presença significativa de assédio moral.

Espera-se que os achados deste estudo possam contribuir pra a introdução de medidas alternativas para redução e enfrentamento do estresse e consequentemente o burnout nos profissionais analisados. A criação de políticas voltadas para a saúde mental deve ser pensada para melhoria da saúde ocupacional dos residentes. A realização de novos estudos com metodologias mais robustas sobre a temática se faz pertinente.

\section{REFERÊNCIAS}

1. Guido LA, Silva LM, Kleinübing RE, Umann J. Stress and coping among surgical unit nurses of a teaching hospital. Rev. RENE. [Internet] 2012 [cited 2019 Feb 10]; 13(2): 428-6. Available from: http://www.periodicos.ufc.br/rene/article/view/3949 
2. Merces MC, Lopes RA, Silva DS, Oliveira DS, Lua I, Mattos AIS et al. Prevalence of burnout syndrome in nursing professional of basic health care. Rev. Fund. Care Online. [Internet] 2017 [cited 2019 Feb 07]; 9(1): 208-14. Available from: http://dx.doi.org/10.9789/2175-5361.2017.v9i1.208-214

3. Rosa SD, Lopes RE. Multiprofessional residency in healthcare and postgraduate education in Brazil: historical remarks. Trab. Educ. Saúde. [Internet] 2009 [cited 2019 May 02]; 7(3): 479-98. DOI: http://dx.doi.org/10.1590/S1981-77462009000300006

4. Guido LA, Silva RM, Goulart CT, Bolzan ME, Lopes LF. Burnout syndrome in multiprofessional residents of a public university. Rev. Esc. Enferm. USP. [Internet] 2012 [cited 2019 May 02]; 46(6): 1477-83. DOI: http://dx.doi.org/10.1590/S008062342012000600027

5. IsHak WW, Lederer S, Mandili C, Nikravesh R, Seligman L, Vasa M, et al. Burnout during residency training: a literature review. J. Grad. Med. Educ. [Internet] 2009 [cited 2019 May 20]; 1(2): 236-242. DOI: https://dx.doi.org/10.4300\%2FJGME-D-09-00054.1

6. Low ZX, Yeo KA, Sharma VK, Leung GK, Mclntyre RS, Guerrero A, et al. Prevalence of Burnout in Medical and Surgical Residents: A Meta-Analysis. Int. J. Environ. Res. Public Health. [Internet] 2019 [cited 2019 Jun 01); 16(9): 1479. DOI: https://doi.org/10.3390/ijerph16091479

7. Abreu KL, Stoll I, Ramos LS, Baumgardt RA, Kristensen CH. Occupational stress and Burnout Syndrome in the professional practice of psychology. Psicol. cienc. prof. [Internet] 2002 [cited 2019 Feb 03]; 22(2): 22-9. Available from: http://pepsic.bvsalud.org/scielo.php?script=sci_abstract\&pid=S1414-98932002000200004\&lng=en\&nrm=iso

8. Grau-Martín A, Suñer-Soler R. Burnout syndrome and medical residents: reflections on the magnitude of the phenomena. Rev. Clin. Esp. [Internet] 2011 [cited 2019 Feb 05]; 211(2): 112-3. DOI: https://doi.org/10.1016/j.rce.2010.06.010

9. Paiva KCM, Gomes MAN, Helal DH. Occupational stress and burnout syndrome: proposition of an integrative model and research perspectives with higher education teachers. Gestão \& Planejament. [Internet] 2015 [cited 2019 Feb 10]; 16(3): 28509. Available from: https://revistas.unifacs.br/index.php/rgb/article/view/3570

10. Freudenberger HJ. Staff Burn-Out. J. Soc. Issues. [Internet] 1974 [cited 2019 May10]; 90(1): 159-65. DOI: https://doi.org/10.1111/j.1540-4560.1974.tb00706.x

11. Maslach C, Jackson SE. The measurement of experienced burnout. J. Organ. Behav. [Internet] 1981 [cited 2019 May 12]; 2: $99-$ 113. DOI: https://doi.org/10.1002/job.4030020205

12. Maslach C. What have we learned about burnout and health? Psychol. Health. [Internet] 2001 [cited 2019 May 15]; 16: 607611. DOI: https://doi.org/10.1080/08870440108405530

13. Ledikwe JH, Kleinman NJ, Mpho M, Mothibedi H, Mawandia S, Semo BW, et al. Associations between healthcare worker participation in workplace wellness activities and job satisfaction, occupational stress and burnout: A cross-sectional study in Botswana. BMJ Open. [Internet] 2018 [cited 2019 Jun 10]; 8(3): e018492. DOI: http://dx.doi.org/10.1136/bmjopen-2017018492

14. Dyrbye LN, Thomas MR, Massie FS, Power DV, Eacker A, Harper W, et al. Burnout and suicidal ideation among U.S. medical students. Ann. Intern. Med. [Internet] 2008 [cited 2019 Jun 10]; 149(5): 334-341. DOI: https://doi.org/10.1002/job.4030020205

15. Maslach C, Jackson S. The measurement of experienced Burnout. J. Occup. Behavior. [Internet] 1981 [cited 2019 Feb 20]; 2(2): 99-113. DOI: https://doi.org/10.1002/job.4030020205

16. Carlotto MS, Palazzo LDS. Factors associated with burnout's syndrome: an epidemiological study of teachers. Cad. Saude Publica. [Internet] 2006 [cited 2019 Feb 20]; 22(5): 1017-26. DOI: http://dx.doi.org/10.1590/S0102-311X2006000500014

17. Maslach C, Jackson SE. Maslach Burnout Inventory. 2a ed. Palo Alto, (CA): Consulting Psychologist Press, 1986.

18. Ramirez AJ, Graham J, Richards MA, Cull A, Gregory WM. Mental health of hospital consultants: the effects of stress and satisfaction at work. Lancet. [Internet] 1996 [cited 2019 Feb 10]; 347(9003): 724-8. DOI: https://doi.org/10.1016/s01406736(96)90077-x

19. World Health Organization (WHO). Obesity: preventing and managing the global epidemic. Geneva: World Health Organization; [Internet] 2000 [cited 2019 Feb 10]. Available from: https://www.who.int/nutrition/publications/obesity/WHO_TRS_894/en/

20. Ferreira LC (Org). A sociologia no horizonte do século XXI. Boitempo: São Paulo; 1997.

21. Antunes R (Org.). A dialética do trabalho: Escritos de Marx e Engels. Expressão Popular: São Paulo; 2004.

22. Goulart CT, Silva RM, Bolzan MEO, Guido LA. Sociodemographic and academic profile of multiprofessional residents of a public university. Rev. Rene. [Internet] 20121996 [cited 2019 Feb 10]; 13(1): 178-86. Available from: http://www.periodicos.ufc.br/index.php/rene/article/view/3792

23. Franco GP, Barros AL, Norgueira-Martins LA, Zeitoun SS. Burnout in nursing residents. Rev. Esc. Enferm. USP. [Internet] 2011 [cited 2019 Feb 20]; 45(1): 12-8. DOI: http://dx.doi.org/10.1590/S0080-62342011000100002

24. Lima PC, Gouveia MTO. Quality of life of health care residents: review. Electronic J. Collection Health. [Internet] 2018 [cited 2019 Feb 20]; 14: s1621-s27. DOI: https://doi.org/10.25248/REAS338_2018

25. Ishak WW, Lederer S, Mandili C, Nikravesh R, Seligman L., Vasa M, et al. Burnout During Residency Training: A Literature Review. J. Grad. Med. Educ. [Internet] 2009 [cited 2019 Apr 10]; 1(2): 236-42. DOI: https://dx.doi.org/10.4300\%2FJGME-D-0900054.1

26. Cavalcanti IL, de Lima FLT, Souza TA, da Silva MJS. Burnout and depression in residents of a Multiprofessional Program in Oncology: a longitudinal prospective study. Rev. bras. educ. med. [Internet] 2018 [cited 2019 Apr 10]; 42(1): 190-8. DOI: http://dx.doi.org/10.1590/1981-52712018v42n1rb20170078

27. Tavares KFA, Souza NVDO, Silva LD, Kestenberg CCF. Prevalence of burnout syndrome among resident nurses. Acta paul. enferm. [Internet] 2014 [cited 2010 Apr 20]; 27(3): 260-65. Available from: http://www.scielo.br/scielo.php?pid=S0103$21002014000300260 \&$ script=sci_arttext\&tIng=en 
28. Guido L, Goulart C, Silva R, Lopes L, Ferreira E. Stress and Burnout among multidisciplinar residents. Rev. Latino-Am. Enfermagem. [Internet] 2012 [cited 2019 Feb 20]; 20(6): 1064-71. DOI: http://dx.doi.org/10.1590/S0104-11692012000600008

29. Jesus MAC, Souza NVDO, Costa CCP, Carvalho EC, Gallasch CH Souza PHDO. Psychological harassment in the hospital nursing workplace: an integrative literature review. Rev. enferm. UERJ. [Internet] 2016 [cited 2019 Jun 10]; 24(4): e26437. DOI: http://dx.doi.org/10.12957/reuerj.2016.26437

30. Lima FD, Buunk AP, Araújo MBJ, Chaves JGM, Muniz DLO, Quiroz LB de. Burnout Syndrome in residents of the Federal University of Uberlândia - 2004. Rev. bras. educ. med. [Internet] 2017 [cited 2019 Feb 20]; 31(2): 137-46. Available from: http://www.scielo.br/scielo.php?script=sci_arttext\&pid=S0100-55022007000200004

31. Moreira AP, Patrizzi L, Accioly M, Shimano S, Walsh I. Quality of life evaluation, sleep and Burnout Syndrome the residentes of the multidisciplinary residency program in health. RMRP. [Internet] 2016 [cited 2019 May 10]; 49(5): 393-02. DOI: https://doi.org/10.11606/issn.2176-7262.v49i5p393-402

32. Rocha SJ, Casarotto AR, Schmitt BAC. Health and Work of the Multiprofessional Residents. Rev. Cienc. Salud. [Internet] 2018 [cited 2019 May 10]; 16(3): 447-62. DOI: http://dx.doi.org/10.12804/revistas.urosario.edu.co/revsalud/a.7265

33. Merces MC, Silva DS, Lua I, Oliveira DS, Souza MC, D’oliveira Júnior A. Burnout syndrome and abdominal adiposity among Primary Health Care nursing professional. Psicol. Reflex. Crit. [Internet] 2016 [cited 2019 May 10]; 29(44): 1-8. DOI: https://doi.org/10.1186/s41155-016-0051-7

34. Rosmond R, Bjorntorp P. Occupational status, cortisol secretory pattern and visceral obesity in middle-aged men. Obes. Research. [Internet] 2000 [cited 2019 May 10]; 8(6): 445-50. DOI: https://doi.org/10.1038/oby.2000.55

35. Oliveira C, Scarabelot VL, Cioto SG, Caumo W, Torres ILS. Interrelation between metabolic syndrome, chronic stress and circadian rhythms of adipogenic markers: a review. Clinical and Biomedical Research. [Internet] 2013 [cited 2019 May 10]; 33(3/4): 257-68. Available from: https://seer.ufrgs.br/hcpa/article/view/39783 